\title{
Rhinocerebral mucormycoses with emphasis on clinical diagnosis, altered host defensive mechanisms and management
}

\author{
DEJA TANPHAICHITRA \\ M.D. \\ Infectious Disease and Host Defense Unit, Department of Medicine, \\ Ramathibodi-Mahidol University Medical Center, Bangkok 4, Thailand
}

\begin{abstract}
Summary
Two cases of malignant form of mucormycosis are presented. In spite of extensive treatment with surgery and antifungal drugs, both cases were fatal. Diminished cellular immunity was demonstrated and considered an important factor in the poor outcome.
\end{abstract}

\section{Introduction}

Malignant rhinocerebral mucormycoses (RCM) are not uncommon in patients with compromised host defences (Eisenberg, Wood and Boles, 1977; Meyer and Armstrong, 1973). Underlying conditions include leukaemia, lymphoma (Meyer, Rosen and Armstrong, 1972), uncontrolled diabetes mellitus (Abramson, Wilson and Arky, 1967), carcinomatosis, renal failure and effects of immunosuppressive therapy.

The progressive nature of the infection in these patients is thought to result from impaired cellular immunity (Tanphaichitra, Limsuwan and Panupornprapongs, 1978). Two patients with RCM are described in whom the outcome was fatal in spite of intensive therapy. E rosette formation and 2,4-dinitrochlorobenzene sensitivity testing indicated impaired cellular immunity.

\section{Case reports}

Case 1

A 30-year-old Thai female with diabetic retinopathy and nephropathy was admitted to hospital with a history of facial pain for 5 days and periorbital swelling for 2 days. The diabetes was controlled with isophane insulin 10-15 u./day which she had been receiving for 2 years, but had stopped during the month before admission.

She was confused, had a mild pyrexia $\left(38^{\circ} \mathrm{C}\right)$ and her face was swollen, warm and tender particularly

Correspondence and requests for reprints: Dr Deja Tanphaichitra, Department of Medicine, Ramathibodi Hospital, Mahidol University Medical Center, Rama 6 Road, Bangkok, Thailand. over the left peri-orbital area. External ocular movement was intact.

Laboratory findings indicated a mild anaemia haemoglobin concentration $9.9 \mathrm{~g} \%$, a leucocytosis of $27.95 \times 10^{9}$ cells $/ 1$ with $95 \%$ polymorphonuclear neutrophils. The blood sugar was $53.8 \mathrm{mmol} / \mathrm{l}$ but no acetone was detected. The blood urea was 23.3 $\mathrm{mmol} / \mathrm{l}$ and creatinine was $698 \mu \mathrm{mol} / \mathrm{l}$.

Her diabetes was controlled with insulin but the confusion persisted. The facial swelling increased with chemosis and total ophthalmoplegia of the left eye. Multiple small facial pustules appeared, vision was lost in the left eye and a blood-stained discharge appeared in the left nasal orifice.

A presumptive diagnosis of RCM was made and confirmed by the demonstration of typical nonseptate hyphae 6-15 $\mu \mathrm{m}$ in diameter in a stained film of scraping from the left nasal septum and turbinate. Cultures were positive for Mucor sp.

Amphotericin B infusions were commenced daily.

An ethmoidectomy was performed revealing necrotic tissues occupying the whole left maxillary antrum and extending into the left ethmoid sinus and nasal cavity. The sinuses were irrigated with amphotericin B.

Microscopic examination of the facial skin lesion showed the presence of fungal hyphae indicating the widespread nature of the infection. On the twentieth day after admission a left total maxillectomy with orbital exentration and excision of left facial skin was performed. The nasal septum turbinate and posterior portion of the hard palate were necrotic. A fungal mass occupied the whole left ethmoid sinus, and involved the left eyeball, parotid gland, buccinator muscle and subcutaneous tissues.

Amphotericin B was continued on an alternate daily regime. An aspiration pneumonia and wound infection was treated with amikacin and clindamycin.

The patient deteriorated and developed signs of intracranial involvement - epileptiform convulsions, 
drowsiness and neck stiffness. The cerebrospinal fluid on the 48th day contained 345 leucocytes $/ \mathrm{mm}^{3}$, $95 \%$ polymorphonuclear neutrophils.

She died on the 50th day. At post-mortem the presence of Mucor sp. was demonstrated in the spinal fluid and extending into the frontal lobe of the left cerebrum.

\section{Case 2}

A 47-year-old male Thai with a stage IV poorly differentiated lymphoma of 8-months' duration. He had been treated with cyclophosphamide, vincristine, prednisone and B.C.G. He also had diabetes mellitus controlled with isophane insulin $15 \mathrm{u}$./day.

He was admitted because of widespread petechial haemorrhages and uncontrollable epistaxis. Physical examination revealed a pale man with petechiae on the trunk and extremities. There was a blood-stained discharge from the right nasal orifice. No ocular abnormalities were observed. There was a general lymphadenopathy with hepatosplenomegaly.

$\mathrm{X}$-rays of the paranasal sinuses revealed a soft tissue mass in the right maxillary antrum.

The blood-stained nasal discharge continued and a right maxillary antrectomy was performed. Necrotic tissue but no pus was found. A scraping of the right nasal turbinate bone revealed fungal hyphae typical of a phycomycete. Rhizopus sp. was cultured.

Amphotericin B was commenced but the infection progressed into the right orbital tissues. The patient died 27 days after admission.

\section{Evaluation of cellular immunity}

The cellular immune status of both patients was assessed by $\mathrm{E}$ rosette formation and 2,4-dinitrochlorobenzene sensitivity.

\section{In vitro}

$\mathrm{E}$ rosette formation: the ability of the $\mathrm{T}$ cells to form rosette spontaneously by binding sheep erythrocytes to their surface was used as a marker for $T$ cells. A leucocyte suspension $0.2 \mathrm{ml}$ containing $10^{6}$ cells was mixed with $0.2 \mathrm{ml}$ of a $0.5 \%$ suspension of the sheep erythrocytes. The mixture was incubated in a water bath at $37^{\circ} \mathrm{C}$ for $5 \mathrm{~min}$. The pellet was then gently resuspended and rosette-forming cells were counted in a cover slip preparation. One hundred lymphocytes were counted and all cells binding 2 or more sheep erythrocytes were considered to be $T$ lymphocytes.

\section{In vivo}

2,4-Dinitrochlorobenzene (DNCB) reaction: the DNCB sensitization procedure consisted on the application of $2000 \mu \mathrm{g}$ of DNCB in $0.1 \mathrm{ml}$ of acetone to an area of skin $2 \mathrm{~cm}$ in diameter on the upper inner portion of the arm. Fourteen days later $100 \mu \mathrm{g}$ of DNCB was applied to the opposite forearm. Subjects were considered sensitized to DNCB if the characteristic sign of delayed hypersensitivity appeared.

\section{Results}

Results of cellular immunity evaluation

\begin{tabular}{lcc}
\hline \multicolumn{1}{c}{ Conditions } & 2,4-DNCB & $\begin{array}{c}\text { E rosette-forming } \\
\text { cells }\end{array}$ \\
\hline Normal (20 cases) & $80 \%$ & $67-81 \%$ \\
Case 1 Mucormycoses & Negative & $42 \%$ \\
Case 2 & Negative & $23 \%$ \\
\hline
\end{tabular}

\section{Discussion}

Zygomycosis (phycomycosis) is the general name given to 2 different entities of fungal infections caused by 2 orders of the class Zygomycetes (Phycomycetes): the Mucorales and the Entomophthorales (Ainsworth, 1973).

Tropical subcutaneous and nasal phycomycosis is a benign chronic infection due to the order Entomophthorales, family Entomophthoraceae. Mucormycosis, a malignant infection of rhinocerebral, pulmonary and abdominal blood vessels is caused by 4 pathogenic families: Cunninghamellaceae, Saksenaeaceae, Mortierellaceae, and Mucoraceae. The family Mucoraceae is the most important one and it includes the genera Absidia, Mucor, and Rhizopus.

The Zygomycetes are ordinarily considered saprophytic except in a compromised host; they commonly invade blood vessels and grow into the lumen, leading to thrombosis, infarction, and provoking a massive polymorphonuclear cell reaction. The clinical diagnosis of RCM depends on characteristic symptoms and signs including facial pain of short duration with periorbital swelling progressing to ptosis, proptosis, loss of corneal reflex and loss of vision. There is frequently a blood-stained nasal discharge with necrosis of the turbinates, paranasal sinus involvement and palatal ulceration.

Confirmation of the diagnosis depends on the demonstration of fungal hyphae in tissue biopsies or mucosal scrapings and the subsequent culture of a Zygomycetes. The differential diagnosis includes staphylococcal and Aspergillus infections and cavernous sinus thrombosis.

Occasionally as in the first case there is a cutaneous involvement (Meyer et al., 1973).

The condition usually has a rapid progress and frequently extends into the cranial cavity with fatal results. Therapy involves management of underlying conditions, surgical excision and the use of specific antifungal agents especially amphotericin $\mathbf{B}$.

The rapidity of progress and the relative ineffectiveness of therapy may be due to impaired cellular 
immunity found in most patients with RCM. The 2 patients reported both showed subnormal $\mathrm{E}$ rosette and DNCB reactions.

Earlier diagnosis, aggressive therapy and the awareness of the impaired cellular immune state may improve the prognosis for patients with RCM.

\section{Acknowledgment}

This study was supported in part by Thai National Research Council.

\section{References}

Abramson, E., Wilson, D. \& ARky, R.A. (1967) Rhinocerebral phycomycosis in association with diabetic ketoacidosis. Annals of Internal Medicine, 66, 735.

AINSWORTH, G.C. (1973) Introduction and keys to higher taxa. In: The Fungi: An Advanced Treatise (Ed. by
Ainsworth, G.C., Sparrow, F.K. and Sussman, A.S.), p. 1 . Academic Press, New York.

EISENBERG, L., Wood, T. \& Boles, R. (1977) Mucormycosis. Laryngoscope, 77, 347.

Meyer, R.D. \& ARMStrong, D. (1973) Mucormycosischanging status. Critical Reviews in Clinical Laboratory Sciences, 4, 421.

Meyer, R.D., Kaplan, M.H., Ong, M. \& Armstrong, D. (1973) Cutaneous lesions in disseminated mucormycosis. Journal of the American Medical Association, 225, 737.

Meyer, R.D, Rosen, P \& Armstrong, D. (1972) Phycomycosis complicating leukemia and lymphoma. Annals of Internal Medicine, 77, 87.

TANPHAICHITRA, D., LIMSUWAN, A. LASSERRE, R., TANPHAICHITRA, V., PanUPORnPRAPONGS, Y. \& TANPhaichitra, P. (1978) Cellular immunity in common intracellular infections in the tropics. In: Chemotherapy of Fungal Diseases. Proceedings of the 10th International Congress of Chemotherapy (1977). (Ed. by Sieganthaler, W. and Lüthy R.). Current Chemotherapy, 1, 53. 